\title{
Surgical treatment of a dissecting aneurysm of the superior cerebellar artery: case report
}

\author{
Florin Stefanescu ${ }^{1}$, Stefanita Dima ${ }^{2}$, Mugurel Petrinel Radoi ${ }^{1}$ \\ National Institute of Neurology and Neurovascular Diseases - Bucharest \\ ${ }^{1}$ Neurosurgical Department \\ ${ }^{2}$ Neuroradiological Department
}

\begin{abstract}
Dissecting aneurysm located in the peripheral region of the superior cerebellar artery is very rare. There is little experience regarding their surgical or endovascular treatment. We present the case of a peripheral dissecting superior cerebellar artery aneurysm treated by surgical clipping.
\end{abstract}

Key words: dissecting peripheral aneurysm, superior cerebellar artery, surgical treatment.

\section{Introduction}

Aneurysms located on the superior cerebellar artery (SCA) are uncommon, accounting for only $0.2 \%$ of all intracranial aneurysms $(11,12,17)$. Few cases of dissecting aneurysms involving the distal portion of the SCA have been previously described $(14,18)$. Due to the rarity of these aneurysms, there is little consensus in the literature about their assessment, treatment and prognosis. Owing the proximity to the vital structures and to the frequent inability to preserve the involved parent artery, surgery of these lesions is challenging. Endovascular therapeutic approach becomes an alternative method of treatment $(3,4)$. Along with a review of the existing literature on this matter, we described our experience with a case of peripheral SCA dissecting aneurysm treated by microsurgical clipping.

\section{Case report}

A 28-year-old woman was admitted to hospital presenting with a 4-day history of headache, dizziness, vomiting and diplopia. She had no relevant medical history. Neurological examination revealed only a right-sided fourth cranial nerve palsy, slight right dismetria and mild nuchal rigidity.

Initial unenhanced cerebral computed tomography (CT) scan showed perimesencephalic and right cerebellopontine angle (CPA) subarachnoid hemorrhage (SAH). Four vessels cerebral angiogram revealed an irregular aneurysm, $3 \mathrm{~mm}$. in diameter, on the right SCA, distal located of the basilar artery, in its cerebellomesencephalic (quadrigeminal) segment (Figure a). 

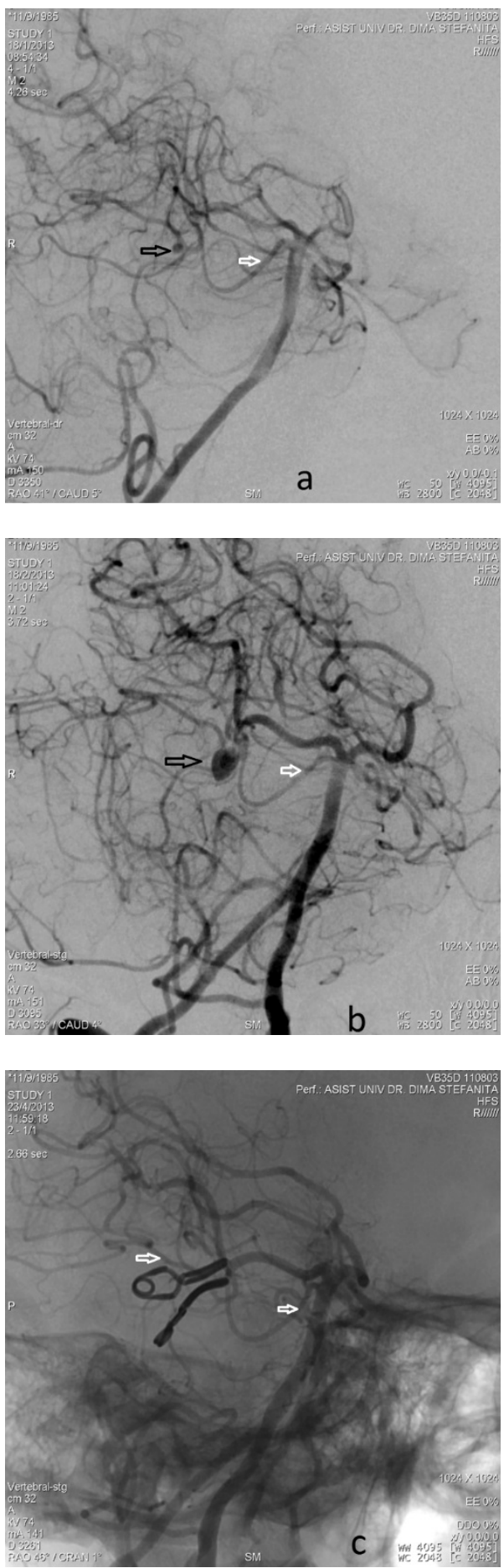

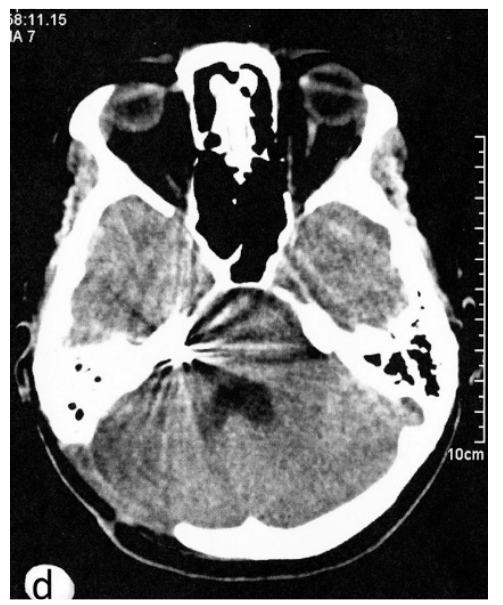

Figure a - Initial cerebral angiographic images showed a $3 \mathrm{~mm}$. irregular aneurysm (black arrow), distally developed on the right SCA (white arrow), in its quadrigeminal segment; Figure $\mathbf{b}$ - Second preoperative cerebral angiography, performed 28 days later, revealed a dissecting, partially thrombosed aneurysm (black arrow), with important changes of the aneurysm's sac, which triple its size $(10 \mathrm{~mm}$.);

Figure $\mathbf{c}$ - postoperative angiographic images demonstrate complete obliteration of the aneurysm with flow preservation in the parent artery (white arrow); Figure d - postoperative native CT scan, performed 2 months after surgery, showed clips

artefacts in the right cerebellopontine angle.

Initially, we considered this lesion as a mycotic peripheral aneurysm and the patient received antifungal therapy (fluconazole, 200 mg once daily). Her condition did not improve continuing to present headache, vomiting and two episodes of loss of consciousness. Rightsided cranial nerve palsy, right dismetria and slurred speech were still present at 4 weeks after the onset of the symptoms. A follow-up cerebral angiogram, performed 28 days after the first one, revealed an important growth of the aneurysms sac, which triple its size measuring $10 \mathrm{~mm}$. in diameter (Figure b). We 
DOI: 10.2478/romneu-2014-0036

considered it as a dissecting aneurysm and proposed surgery as method of treatment.

We approached the aneurysm through a right lateral suboccipital craniotomy. The aneurysm was located distally on the SCA, 25 $\mathrm{mm}$ from basilar artery, behind the brainstem perforating arteries. It was exposed on the lateral aspect of the brainstem along with the parent artery. After aneurysm reconstruction and without intra-operative complications, two curved aneurysm clips were applied to the neck in parallel to the SCA main trunk, preserving its patency. Postoperative angiography showed complete exclusion of the aneurysm with flow preservation in the distal right SCA (Figure c)

Postoperative period was uneventful and no additional deficits were noted. The patient was discharged complaining only of mild diplopia, blurred vision and dizziness. Six months after surgery the patient was in good general condition, without neurologic motor deficits and complete improvement of diplopia and blurred vision. Non-contrast CT scan showed a normal postoperative aspect with clips artefacts in the right cerebellopontine angle (Figure d).

\section{Discussion}

The overall incidence of peripherally located aneurysms in the anterior cerebral artery distribution is between $7 \%$ and $9 \%$, followed by $2 \%-7 \%$ for the middle cerebral artery location, and only $5 \%$ for the posterior circulation $(8,15)$. Usually, superior cerebellar artery aneurysms manifest with subarachnoid hemorrhage $(\mathrm{SAH})$. A peripheral ruptured aneurysm of the superior cerebellar artery
(SCA) should be considered when SAH predominates in perimesencephalic and superior cerebellar cisterns (6). Although many of these lesions are saccular, fusiform and dissecting aneurysms are not unusual (1, 9 , 13, 17). Despite being a distal lesion, a mycotic origin has seldom been proven in SCA aneurysm $(9,13)$. When presenting with $\mathrm{SAH}$, the diagnosis of a dissecting aneurysm of the SCA can be difficult, as in our case, requiring serial angiograms to demonstrate progressive change in affected vascular segments.

Distal SCA aneurysms may have distinctive clinical features in accordance with their location. The trochlear nerve, located beneath the tentorium, is vulnerable to injuries related to aneurysms involving the nearby posterior cerebral artery (P2 and P3 segments) or to SCA $(4,5,17)$.

According to a general consensus on SCA aneurysms, the presence of important perforating arteries and adjacent cranial nerves makes clipping of the SCA aneurysms a very challenging surgery. Many complications are related to lower cranial nerve dysfunction because of the intimate relationship between these aneurysms and cranial nerves $(10,14)$. In the case of dissecting SCA aneurysms surgical options include proximal occlusion, trapping, clip reconstruction and distal outflow occlusion $(14,15)$. In any event, care should be taken to preserve the parent artery and ventral brainstem perforating branches, and to mitigate the risk of brainstem infarction. When proximal occlusion or trapping are used, it is advisable to use combined distal revascularization techniques to prevent permanent ischemic damage to the brainstem 
and cerebellar hemisphere due to unpredictable collateral supply $(14,15,16)$.

The surgical approach depends on the site of the aneurysm. Aneurysm of the cisternal segments may be treated by the pterional transsylvian, subtemporal transtentorial or occipital transtentorial approach, whereas aneurysms of the cortical segments are usually clipped through suboccipital approach for the marginal branch, infratentorial supracerebellar or occipital transtentorial approach for the hemispheric branch, and suboccipital, infratentorial supracerebellar or occipital transtentorial approach for the vermian branch $(18,19)$. In our case, we preferred the lateral suboccipital approach because angiographic findings suggested that the aneurysm was partially trombosed indicating a larger lesion and it was located more distally. Dissection was carried out over the cerebellar hemisphere and the relationship between the brainstem and aneurysm was considered without difficulty.

The endovascular therapy may be a good alternative for the treatment of SCA aneurysms. Endovascular occlusion with detachable coils is feasible with good angiographic and clinical results and with low morbidity $(3,7)$. Endovascular option includes coil embolization of narrow, mostly saccular aneurysms and stent-supported coil placement for wider, fusiform aneurysms (2).

\section{Conclusions}

Peripheral, dissecting aneurysms of the SCA are rare and there is little consensus over their surgical or endovascular management. When endovascular occlusion of the parent artery is performed the risk of the ischemic injury should always be considered. We presented the case of a peripheral SCA aneurysms which had radiological features suggestive of dissecting lesions, and considered that an experienced neurovascular surgeon team could clip the aneurysm with parent artery preservation.

\section{Correspondence}

Mugurel Petrinel Radoi MD, PhD

Affiliation: National Institute of Neurology and

Neurovascular Diseases - Bucharest,

Neurosurgical Department,

Postal Address: National Institute of Neurology and

Neurovascular Diseases - Bucharest,

Neurosurgical Department, 10-12 Berceni str.,

041902 Bucharest, Romania

E-mail address: muguradoi@yahoo.com

Telephone: 0040723527292

\section{References}

1. Atalay B, Altinors N, Yilmaz C, Caner H, Ozger O. Fusiform aneurysm of the superior cerebellar artery: short review article. Acta Neurochir (Wien) 149: 291-294, 2007.

2. Cardoso CA, Almeida Lima EL, Barbosa TM. Dissecting aneurysm of the distal superior cerebellar artery - Case report and assessment of endovascular treatment. Arq Bras Neurocir 32(2):118-21, 2013.

3. Chaloupka JC, Putman CM, Awad IA. Endovascular therapeutic approach to peripheral aneurysms of the cerebellar artery. AJNR Am J Neuroradiol. 1996; 17(7):1338-42.

4. Danet M, Raymond J, Roy D. Distall superior cerebellar artery aneurysm presenting with cerebellar infarction: report of two cases. AJNR Am J Neuroradiol 22:717-720, 2001.

5. Drake CG. The treatment of aneurysms of the posterior circulation. Clin Neurosurg 26:96-144, 1979

6. Gacs G, Vinuela F, Fox AJ, Drake CG. Peripheral aneurysms of the cerebellar arteries. J Neurosurg 1983;58:63-8. 
DOI: 10.2478/romneu-2014-0036

7. Haw C, Willinsky R, Agid R, TerBrugge K. The endovascular management of superior cerebellar artery aneurysms. Can J Neurol Sci. 31(1):53-7, 2004.

8. Kubota S, Ohmori S, Tatara N, Nagashima C. A ruptured peripheral, superior cerebellar artery aneurysm: A case report and a review of the literature as to surgical approaches. No Shinkei Geka 1994;22:279-83.

9. Kurosu A, Fujii T, Ono G. Distal superior cerebellar artery aneurysm. Br J Neurosurg 14:244-246, 2000.

10. Lubicz B, Leclerc X, Gauvrit JY, Lejeune JP, Pruvo JP. Endovascular treatment of peripheral cerebellar artery aneurysms. AJNR Am J Neuroradiol 24(6): 1208-1213, 2003.

11. Matricalli B, Seminara P. Aneurysm arising from the medial branch of the superior cerebellar artery. Neurosurgery. 1986;18 (3):350-2.

12. Murtagh F, Balis GA. CT localization of posterior fossa bleeding sites in subarachnoid hemorrhage. Surg Neurol. 1981; 16:211-7.

13. Nakai Y, Hyodo A, Yanaka K, Akutsu H, Nose T. Distal superior cerebellar artery aneurysm in a patient with systemic lupus erythematosus: case report. Surg Neurol 54:73-76, 2000.

14. Nussbaum ES, DefilloA, Zelensky A, stoller R, Nussbaum L. Dissecting peripheral superior cerebellar aneurysms: Report of two cases and review of the literature. Surg Neurol Int. 2011; 2:69.

15. Nussbaum ES, Madison MT, Goddard JK, Lassig JP, Nussbaum LA. Peripheral intracranial aneurysms: Management challenges in 60 consecutive cases. J Neurosurg 2008;109:23-7.

16. Nussbaum ES, Madison MT, Myers ME, Goddard JK, Janjua TM. Dissecting aneurysms of the posterior inferior cerebellar artery: Retrospective evaluation of management and extended follow-up review in 6 patients. J Neurosurg 2008; 109:23-7.

17. Peluso JIP, van Rooij WJ, Sluzewsky M, Beute GN. Distal aneurysms of cerebellar arteries: incidence, clinical, presentation, and outcome of endovascular parent vessel occlusion. AJNR Am J Neuroradiol 28:1573-1578, 2007.

18. Sato M, Kodama N, Sasaki T, Watanabe Z. Aneurysms arising from the cortical segment of the superior cerebellar artery - two case reports. Neurol Med Chir (Tokyo). 39:858-862, 1999.

19. Yasargil MG (ed). Distal Superior Cerebellar Aneurysms, in: Microneurosurgery II, New York: Thieme 1984: 279-280. 